\title{
NEW CRISPR/CAS9 GENE EDITING TECHNOLOGY FOR DEVELOPMENT OF AGRICULTURAL BIOTECHNOLOGY
}

\author{
O.I. Kershanskaya ${ }^{l}$, Zh. Kuli ${ }^{1}$, A. Maulenbay ${ }^{1}$, D. Nelidova ${ }^{l}$, S.N.
${ }^{1}$ Institute Plant Biology and Biotechnology, Almaty, Kazakhstan
${ }^{2}$ The James Hutton Institute, Dundee, Scotland, UK, gen_o.kersh@mail.ru
}

\begin{abstract}
Plant resistance is an economical and sustainable stress management option. For plant diseases complete resistance does not exist and partial resistance has mostly been ineffective under field conditions. Our efforts to increase the strength of the plant innate defense system using the best modern gene editing technology CRISPR/Cas9 in combination with elaboration of simple and effective method of germ-line genetic transformation would be effective to limit viral and fungal pathogens in barley and to produce yield potential elite barley varieties with high economical benefice.

Keywords: CRISPR/Cas9, genome editing, stress resistance, barley
\end{abstract}

DOI: $10.31255 / 978-5-94797-319-8-1434-1437$

Introduction: The technology, a genome-editing tool called CRISPR-Cas9, revolutionized the life sciences when it appeared on the market in 2013 [Doudna, Charpentier, 2013, 2014; Le Cong et al., 2013]. CRISPR (Clustered Regularly Interspaced Short Palindromic Repeats) gene-editing is allowing rapid scientific advances in many fields, including human health and now it has been shown that crop research can also benefit from this latest exciting technology. New study of CRISPR-Cas9 technology shows potential to improve crop efficiency [Feng et al., 2013, 2014; Nekrasov et al., 2013]. It is now proving useful in the plant science community as a powerful tool for the improvement of agricultural crops. It is estimated that up to $40 \%$ of harvest is lost worldwide to pests/diseases threatening our food supply. Barley is susceptible to many viral and fungal diseases that can result in drastically reduced yield and poor quality grain. Barley cultivars with improved resistance to fungal or viral pathogens will benefit farmers and the local economy by increasing harvest yields and grain quality. The main goals are to elaborate and evaluate new germ-line transformation technology for delivering both conventional and CRISPR/Cas9-based constructions into barley zygote, and new gene editing CRISPR/Cas9 technology for creation of elite fungal and viral diseases resistant barley cultivars.

Materials: 5 UK barley cultivars (Tipple, Bowman, Ovation, Origin and Optic) and 5 KZ barley cultivars (Asem, Inkar, Sari-Arua, Arna, Saule). Methods: CRISPR/Cas 9: What is it and how does it work? CRISPR stands for Clustered Regularly Interspaced Short Palindromic Repeats. The discovery of the type II prokaryotic CRISPR "immune system" has allowed for the development for an RNA-guided genome editing tool that is simple, easy and quick to implement. The CRISPR/Cas9 system consists of a single monomeric protein and a chimeric RNA. A 20-nt sequence in the gRNA confers sequence specificity and cleavage is mediated by the Cas9 protein. Watson-Crick base pairing with the target DNA sequence is the basis for gRNA-based cleavage, making sophisticated protein engineering for each target unnecessary. Only a $20 \mathrm{nt}$ in the gRNA is needs to be modified to facilitate the recognize a different target.

In collaboration with JHI, UK, proposed approach to barley diseases combat are included elaboration of new modern the most effective technique of CRISPR-Cas9 gene editing [Barakate et al., 2014, 2016]; methods of molecular genes cloning and constructing of key anti-fungal and anti-viral constructs; establishment of new Agrobacterium - mediated germ-line genetic transformation biotechnology; creation an effective binary vector for $\boldsymbol{A c}$ and glucanase genes transformation, and knock out the eukaryotic translation initiation factor 
(eIF4E) that is required by many viruses for multiplication; screening and molecular detection of transgenes and editing events by PCR and RT-PCR analysis; testing of created barley plants on micropathogenes and viruses resistance. Expected results: 1. New gene editing CRISPR-Cas9 biotechnology for creation of new elite viral disease resistant barley cultivars. 2. New germ-line transformation biotechnology for delivery of CRISPR-Cas9and valuable genetic constructs into barley. 3. Constructs of chitin ac and glucanase genes for barley transformation. 4. CRISPR construct to knock out the eukaryotic translation initiation factor (eIF4E) for viruses multiplication. 5. Segregation away from the edited event the transformation cassette including Cas9 and guide RNA to leave a 'clean' not GMO mutation. 6. Production of genome edited barley plants with resistance to viral and fungal diseases.

Results and discussion: Plant CRISPR/Cas9 products are intended for Agrobacteriummediated plant transformation or biolistic microparticle bombardment or protoplast transformation. RNAi silencing of eIF4E has conferred resistance to multiple viruses in melon and broad spectrum resistance to potyviruses in tomato. More recently, Arabidopsis complete resistance to Turnip Mosaic Virus has been successfully engineered by editing eIF4E using the CRISPR/Cas9 tool. The products are based on the type IIA CRISPR/Cas9 derived from Streptococcus pyogenes. CRISPR/Cas9 Plasmids for use in plants have been shown in figure.

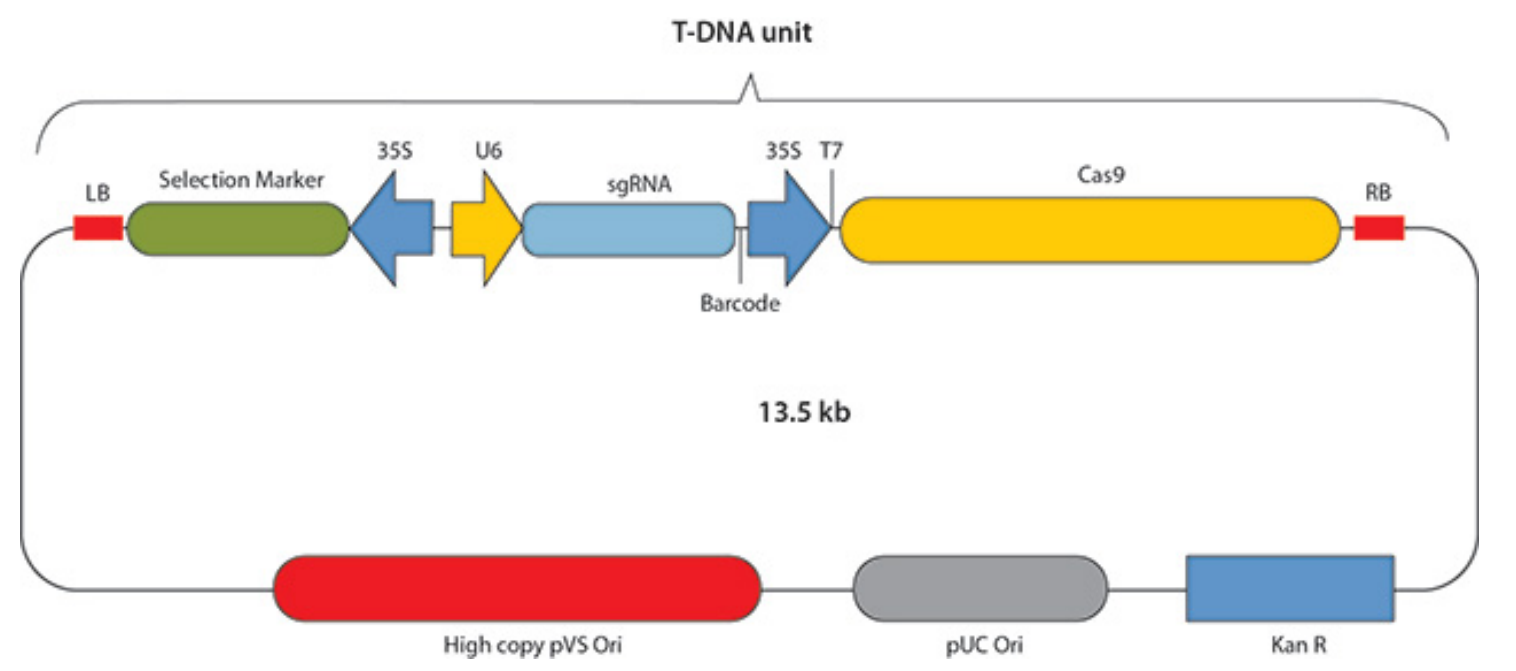

Figure. Scheme of T-DNA unit for monocot CRISPR/Cas9 editing.

The native Cas9 coding sequence was codon optimized for expression in monocots and dicots, respectively. The monocot Cas9 constructs contain a monocot U6 promoter for sgRNA expression. The plant selection markers include hygromycin B resistance gene, neomycin phosphotransferase gene, and the bar gene (phosphinothricin acetyl transferase).

CRISPR/Cas9 Genome Editing Tools include: 1. Design sgRNA. Requires the identification of target sites with specific sequence criteria, while also avoiding the potential for off-target effects. 2. Transcribe and screen sgRNA in vitro. Quickly transcribe any sgRNA in vitro at high yields, without ligation. Don't waste time delivering ineffective sgRNAs to cells - test the cleavage efficiencies of individual sgRNAsin vitro before performing gene editing in your target cells. 3. Deliver sgRNAs and Cas9 into cell. There are several options for delivering sgRNAs and Cas9 to your target cells: for plasmid delivery, Cas9/sgRNA coexpression vectors allow seamless insertion of sgRNAs, and express bright fluorescent markers. Use gesicles, which are cell-derived nanovesicles, for efficient delivery of active Cas9 ribonucleoprotein (RNP) complexes to a broad range of cell types with reduced offtarget effects and very low cytotoxicity. To prevent genomic integration of Cas9, using this 
single reagent to transfect target cells with Cas9 mRNA and sgRNAs without cytotoxic effects. This AAV2-based system for delivery of sgRNAs and Cas9 enables efficient gene editing in difficult-to-transfect cells without genomic integration of Cas9. 4. For sgRNAs and Cas9 delivering into barley, we are using our elaborated A. tumefaciens - mediated pollen germ - line transformation technique. 5. Detection Cas9 protein and confirmation gene editing. Confirm that Cas 9 protein is being expressed in target cells. Ensure that your cell population contains mutations at your target locus by using a mismatch detection assay that outperforms a CEL-1 based assay. 6. Genotype determination. If there are indels on one or both copies of your target gene, Cas9/sgRNA-mediated in vitro cleavage reaction can accurately determine cell's genotype after gene editing. 7. Identity indels. Characterize CRISPR/Cas9-induced indels with a simple four-step protocol using the Guide-it Indel Identification Kit.T1 seeds should be germinated on antibiotic selection and confirmed as transgenic by PCR. Multiple seed from each independent line will be grown up as replicates and leaf tissue or whole plants will be used for assays with fungal spores (KZ) or virus isolates (UK) to determine resistance levels.

CRISPR/Cas9 consists of a Cas9 protein, a CRISPR RNA (crRNA), and a transactivating crRNA (tracrRNA). In gene editing applications, crRNA and tracrRNA are often fused into a single guide RNA (sgRNA). The ribonucleoprotein invades the target with crRNA guide sequence by forming a 20-bp RNA/DNA hybrid and displacing the opposite DNA strand after it encounters a protospacer adjacent motif (PAM), such as NGG. Cas9 endonuclease subsequently cleaves the complementary DNA strand (target strand) with a $\mathrm{HNH}$ nuclease domain and the displaced DNA strand (non-target strand) with a RuvC-like nuclease domain to create a double strand break (DSB). The repair of the DSB by host cell via non-homologous end joining (NHEJ) or homology directed repair (HDR) pathways can be utilized to create gene knockout or introduce a specific genetic modification through homologous recombination with a DNA donor.

RNA-guided endonucleases (RGENs) consisting of the Cas9 protein derived from Streptococcus pyogenes and guide RNAs (gRNAs) can be customized by replacing only the RNA component leading to decreases in labor and time compared to other gene editing methods. Using either Agrobacterium tumefaciens or by trans-fecting plasmids that encode them, programmable nucleases can be delivered into plant cells, where these nucleases cleave chromosomal target sites in a sequence-dependent manner. The result is site-specific DNA double-strand breaks (DSBs) whose repair by endogenous systems results in targeted genome modifications. Main advantages of CRISPR/Cas9 are in terms of simplicity, accessibility, cost and versatility.

The classification of genome edited plants is currently under review to decide whether new breeding technologies including CRISPR/Cas9 are exempt from GM classification. As known RNAi silencing of eIF4E has conferred resistance to multiple viruses in melon and broad spectrum resistance to potyviruses in tomato. More recently, Arabidopsis complete resistance to Turnip Mosaic Virus has been successfully engineered by editing eIF4E using the CRISPR/Cas9 tool. Once the gene edited events are established in barley, the transformation cassette including Cas9 and guide RNA was segregated away from the edited event to leave a 'clean' mutation. The fungal cell wall is composed of chitin, glucans and other polymers. Homologs of both these genes $\boldsymbol{A c}$ gene from Amaranthus caudatus have been overexpressed in a number of species and have shown various effects on fungal resistance. By targeting these main structural components we hoped to break down the fungal cell wall to prevent fungal attack. It has also been shown that enhancing glucanase activity in barley improves its quality as feed for livestock. Traditionally, barley-fed poultry have poor growth rates because they are deficient in glucanases and cannot fully break down endosperm cell walls. Feeding studies in literature have shown that poultry fed genetically modified barley 
with heat stable glucanase outperformed poultry that were fed conventional barley. Introducing gene sequences using the CRISPR/Cas9-based technology is still inefficient so we used binary vectors containing a chitinase gene $\boldsymbol{A c}$ and a heat stable glucanase gene for co-transformation experiments in barley. T1 seeds were germinated on antibiotic selection and confirmed as transgenic by PCR. Multiple seed from each independent barley line grown up as replicates and leaf tissue or whole plants used for assays with fungal spores (KZ) or virus isolates (UK) to determine resistance levels.

Conclusions: The genome edited plants with fungus and virus resistance could be produced. These new technologies will provide the means to target other quality traits (including agronomic and malting) for improved barley cultivars in the future. The development of a new genotype-independent, plant friendly transformation protocol in barley will be interested to plant scientists enabling future experiments to be done in elite cultivars rather than the only transformable Golden Promise cultivar which is of little commercial importance. Once the gene edited events are established in barley, the transformation cassette including Cas9 and guide RNA will be segregated away from the edited event to leave a 'clean' mutation. Edited plants could be segregated to produce 'clean' plants free of vector sequence which may be classified as GMO-free and suitable for future planting.

\section{References}

Barakate A., Higgins J.D., Vivera S., Stephens J., Perry R.M., Ramsay L., Colas I., Oakey H., Waugh R., Franklin F.C.H., Armstrong S.J., Halpin C. The synaptonemal complex protein ZYP1 is required for imposition of meiotic crossovers in barley // The Plant cell. 2014.

Barakate A., Stephens J. An overview of CRISPR-based tools and their Improvements: new opportunities in understanding plant-pathogen interactions for better crop protection // Front. Plant Sci. - 2016. - https://doi.org/10.3389/fpls.2016.00765.

Doudna J.A., Charpentier E. The new frontier of genome engineering with CRISPRCas9 // Science. - 2014. - V. 346 (6213). - P. 1258096.

Feng Z., Mao Y., Xu N., Zhang B., Wei P., Yang D.L. et al. Multigeneration analysis reveals the inheritance, specificity, and patterns of CRISPR/Cas-induced gene modifications in Arabidopsis // Proc. Nat. Acad. Sci. USA. - 2014. - V. 111. - P. 4632-4637.

Feng Z., Zhang B., Ding W., Liu X., Yang DL., Wei P., Cao F., Zhu S., Zhang F., Mao Y., Zhu J.K. Efficient genome editing in plants using a CRISPR/Cas system // Cell Res. 2013. - V. 23. - P. 1229-1232.

Le Cong F., Ran A., David C., Lin Sh., Barretto R., Habib N., Hsu PD., Wu X., Jiang W., Marraffini L.A., Feng Z. Multiplex genome engineering using CRISPR/Cas systems // Science. - 2013. - V. 339, I. 6121. - P. 819-823.

Nekrasov V., Staskawicz B., Weigel D., Jones J.D., Kamoun S. Targeted mutagenesis in the model plant Nicotiana benthamiana using Cas9 RNA-guided endonuclease // Nat. Biotechnol. - 2013. - V. 31. - P. 691-693. 